\title{
Persistent Neutrophilic Meningitis in an Immunocompetent Patient after Basilar Skull Fracture: Case Report
}

\author{
Jaime S Green ${ }^{*}$, Shira R Abeles ${ }^{1}$, Daniel Z Uslan² and Sanjay R Mehta ${ }^{1}$
}

\begin{abstract}
Background: Persistent neutrophilic meningitis is an unusual form of chronic meningitis that is defined as clinical meningitis with a neutrophilic pleocytosis that persists for greater than 7 days despite empiric antimicrobial therapy. Although numerous disease processes can cause this syndrome, the majority of cases are due to opportunistic pathogens infecting immunocompromised hosts.

Case Presentation: A 47 year-old female presented after basilar skull fracture with persistent neutrophilic meningitis unresponsive to empiric broad-spectrum antibiotics. After more than weeks of intensive therapy, 4 hospitalizations and 3 relapses, Nocardia cyriacigeorgica was identified from cerebral spinal fluid. Induction therapy was begun with Ceftriaxone and trimethoprim-sulfamethoxazole (TMP-SMX) for 6 weeks followed by therapy with TMP-SMX and doxycycline for one year. The patient made a complete recovery without sequelae.

Conclusions: Due to the difficulty in obtaining a microbiologic diagnosis, appropriate treatment in cases of persistent neutrophilic meningitis is often delayed leading to morbidity, This case highlights a number of the unique features of Nocardia meningitis and the importance of considering Nocardia infection as a cause of persistent neutrophilic meningitis even in immunocompetent patients.
\end{abstract}

\section{Background}

Persistent neutrophilic meningitis is a syndrome defined by: clinical meningitis (headache, altered sensorium, fever and/or meningismus) with neutrophilic cerebrospinal fluid (CSF) pleocytosis (differential containing $>50 \%$ neutrophils), elevated protein and hypoglycorrhachia that persists for greater than 7 days despite appropriate empiric antimicrobial treatment [1]. Numerous infectious and non-infectious etiologies have been identified as causes of this syndrome including: saprophytic intracellular bacteria (eg. Nocardia, Actinomyces and Brucella), mycobacteria, fungi (eg. Candida spp., Aspergillus spp., zygomycetes, endemic fungi), connective tissue disease (eg. Lupus, adult Still's disease [2], central nervous system (CNS) vasculitides), chemical meningitis, intracranial tumors (eg. craniopharyngiomas), and hypersensitivity reactions $[1,3]$. In the majority of cases of persistent

\footnotetext{
* Correspondence: jsgreen@ucsd.edu

'Department of Medicine, Division of Infectious Diseases, University of California, San Diego, California, USA

Full list of author information is available at the end of the article
}

neutrophilic meningitis due to an infectious etiology, the syndrome is part of a multi-system disseminated process usually in an immunocompromised individual. The atypical nature of many of the organisms associated with this syndrome leads to difficulty in obtaining microbiologic diagnoses, and the etiologic agent is often presumed from epidemiologic risk factors, indirect microbiologic evidence or made postmortem $[1,3]$. Thus, the choice of empiric antibiotic treatment regimen can be a challenging clinical decision. Here we present a case of persistent neutrophilic meningitis in an immunocompetent individual following basilar skull fracture.

\section{Case Presentation}

Hospitalization 1 (basal skull fracture)

A 47 year-old healthy female sustained a ground level fall from a stepladder in her garage without loss of consciousness. The fall resulted in a basilar skull fracture complicated by a small subdural hematoma. She quickly recovered with conservative management and was discharged from the hospital within 4 days.
Ciomed Central

() 2011 Green et al; licensee BioMed Central Ltd. This is an Open Access article distributed under the terms of the Creative Commons Attribution License (http://creativecommons.org/licenses/by/2.0), which permits unrestricted use, distribution, and reproduction in any medium, provided the original work is properly cited. 
Hospitalization 2a (acute meningitis at outside facility) One week after her fall, she represented to an outside hospital with a fever of $102^{\circ} \mathrm{F}$, headache, and altered mental status. Broad-spectrum antibiotics were initiated and after several days a lumbar puncture (LP) was performed. Analysis of the CSF was notable for an opening pressure of $33 \mathrm{~cm}$ of $\mathrm{H}_{2} \mathrm{O}, 3600$ white blood cells (WBC) per deciliter with a neutrophilic predominance (Hospitalization 2a Table 1). CSF gram stain and cultures were obtained which demonstrated no organisms or growth, respectively. The patient eventually required a lumbar drain to manage persistently high intracranial pressures. A CT scan of the head and an MRI of the brain and spine revealed a small subdural hematoma without evidence of a para-spinal or para-meningeal focus of infection. Repeat analyses of the CSF demonstrated negative gram and silver stains, negative direct antigen testing for crytpococcus, Hemophilus influenzae, Influenza A and B, Neisseria meningitidis, Streptococcus agalactiae and Streptococcus pneumoniae; as well as bacterial and fungal cultures with no growth. Serologic testing for coccidiodimycosis, HIV and anti-nuclear antibody testing were negative. While on vancomycin and ceftazidime (14 days), she continued to have daily fevers associated with severe headaches, nausea, photophobia and neck stiffness. Due to a persistence of symptoms, ceftazidime was changed to meropenem and therapy continued for 14 additional days. A short period of defervescence occurred after the initiation of meropenem, but then the fever quickly returned while still on therapy. The patient was transferred to our facility for a second opinion.

\section{Hospitalization $2 b$ (persistent neutrophilic meningitis, transfer to our facility)}

On evaluation at our institution the patient was ill appearing and nauseated with a severe frontal headache that radiated to the occiput. Physical exam was notable for a temperature of $102^{\circ} \mathrm{F}$ and meningismus with an otherwise normal neurologic exam. A sutured laceration on the occipital region of the patient's head was noted to be moderately tender, but without fluctuance or drainage. Serially sampled CSF continued to have a predominantly neutrophilic pleocytosis (Hospitalization 2b Table 1). General labs were normal and repeated microbiologic studies were nondiagnostic. Broad-spectrum antibiotics were resumed (vancomycin and meropenem), however the patient continued to have high daily fevers. High dose trimethoprim-sulfamethoxazole (TMP-SMX) was added on the $8^{\text {th }}$ hospital day. An MRI of the brain and spine was notable for opacification of the left sphenoid sinus and mild pachymeninigitis at the occipital and anterior aspects of the brain; sites where coup and contrecoup injuries could be expected following the patient's fall. No focus of infection was identified. A non-displaced left lateral wall sphenoid sinus fracture and occipital bone fracture were noted on CT sinus scan. A follow up CT cisternogram was negative for any CSF leak. Upon endoscopic ethmoid and sphenoid sinus visualization, a sphenoid sinus hematoma was identified and removed. Bacterial, mycobacterial and fungal cultures of this sampled material remained negative for any growth. The patient's clinical course improved, and after six weeks of vancomycin and meropenem (including five days of TMP-SMX) antibiotics were serially discontinued and the patient was discharged home without an etiologic diagnosis.

\section{Hospitalization 3 (re-admission neutrophilic meningitis)}

Nine days after discharge, the patient represented to our institution with fevers, headaches and lateral gaze ophthalmoplegia. Repeat LP and imaging studies were performed. The previously noted pachymeningitis and opacification of left sphenoid sinus and left occipital bone fracture were noted with no new findings. A tagged white blood cell (WBC) scan utilizing Indium-111 labeled WBC was also unrevealing. The patient underwent repeat endoscopic surgery with application of fibrin glue to the left sphenoid sinus (where the hematoma had been previously removed). During this procedure a pressurized

Table 1 CSF analysis from the four hospitalizations noting neutrophilic predominance

\begin{tabular}{|c|c|c|c|c|c|}
\hline & WBCs (cells $/ \mathrm{mm}^{3}$ ) & Neutrophils (\% of WBCs) & Lymphocytes (\% of WBCs) & Glucose $(\mathrm{mg} / \mathrm{dL})$ & Total Protein $(\mathrm{mg} / \mathrm{dL})$ \\
\hline \multicolumn{6}{|l|}{ Hospitalization $2 \mathrm{a}$} \\
\hline $12 / 21$ & 3600 & 83 & & 32 & 33 \\
\hline \multicolumn{6}{|l|}{ Hospitalization $2 \mathrm{~b}$} \\
\hline $1 / 8$ & 1850 & 83 & 11 & 21 & 90 \\
\hline $1 / 13$ & 2100 & 77 & 15 & 24 & 99 \\
\hline \multicolumn{6}{|l|}{ Hospitalization 3} \\
\hline $2 / 6$ & 520 & 64 & 24 & 26 & 101 \\
\hline $2 / 14$ & 396 & 94 & 4 & 13 & 161 \\
\hline Hospitalization 4 & 480 & 83 & 14 & 17 & \\
\hline
\end{tabular}

CSF = cerebral spinal fluid, $\mathrm{mg} / \mathrm{dl}=$ milligram/deciliter, $\mathrm{WBC} s=$ white blood cells. 
fluorescein CSF dye study was performed which did not show any evidence of a CSF leak. The patient was treated with 14 days of meropenem and TMP-SMX therapy, and discharged home with clinical improvement, but no microbiologic diagnosis.

\section{Hospitalization 4 (re-admission neutrophilic meningitis)}

Eleven days after completing meropenem and TMPSMX, she once again developed fevers and headaches requiring a $4^{\text {th }}$ hospitalization at another institution. Eight weeks after sampling, the CSF culture from the 3rd hospitalization grew Nocardia spp. that eventually was identified as Nocardia cyriacigeorgica complex. The same organism was concomitantly recovered from a lumbar puncture performed during the $4^{\text {th }}$ hospitalization. The isolate was found to be sensitive to ceftriaxone, imipenem, linezolid, doxycycline, moxifloxacin and trimethoprim-sulfamethoxazole (Table 2). Ceftriaxone 2g IV daily and trimethoprim-sulfamethoxazole 2 DS BID were initiated after which the fevers, headaches and ophthalmoplegia resolved. The induction course of therapy was continued for 6 weeks followed by maintenance therapy with TMP-SMX 2 DS BID and doxycycline $100 \mathrm{mg}$ po bid for 1 year. As of the writing of this manuscript she is greater than 12 months post completion of therapy and remains asymptomatic.

Nocardia is a gram-positive actinomycete that is a ubiquitous saprophyte found in the environment (soil, dust, decaying vegetation) [4]. It causes a wide spectrum of disease in humans depending on the immune status of the host. Primarily, it is an opportunistic pathogen that affects patients with impaired cell mediated immunity [5]. Infection with Nocardia most commonly presents as a pulmonary syndrome with nodules, necrosis and/or cavities in $77 \%$ of cases. Central nervous system infection in the form of brain abscesses [6] and occasionally meningitis $[7,8]$ typically occurs after dissemination. In immunocompetent individuals, direct skin inoculation can result in skin and soft-tissue infections. Presentations of this form of nocardiosis include mycetoma, sporotrichoid infections and occasionally osteomyelitis $[9,10]$.

Table 2 Sensitivity of Nocardia cryiacigeorgica isolated from the CSF

\begin{tabular}{|c|c|c|}
\hline Organism: & Antibiotic & $\begin{array}{l}\text { Sensitivity (MIC in mcg/ } \\
\text { ml) }\end{array}$ \\
\hline Nocardia cryiacigeorgica & Ceftriaxone & $1.2(S)$ \\
\hline \multirow{5}{*}{$\begin{array}{l}\text {-Isolated from CSF 2/ } \\
14\end{array}$} & Doxycycline & $.6(S)$ \\
\hline & Imipenem & $.3(\mathrm{~S})$ \\
\hline & Linezolid & $.6(S)$ \\
\hline & Moxifloxacin & $2.5(S)$ \\
\hline & Trim/Sulfa & $.3(\mathrm{~S})$ \\
\hline
\end{tabular}

CSF = cerebral spinal fluid.
Persistent neutrophilic meningitis is different from the usual forms of chronic meningitis that typically begin as a neutrophilic process but then evolve to a lymphocytic predominance. Several unique features of Nocardia enable neutrophilic persistence. High levels of catalase and superoxide dismutase produced by Nocardia species neutralize the killing capacity of neutrophils. T-cells and macrophages thus play an important role in clearing Nocardia infection, while neutrophils mainly act to inhibit filament formation and growth of the bacterium [11-13], The short half-life of the neutrophil requires continued neutrophil recruitment to maintain inhibitory effects on the pathogen's growth, which drives abscess formation [13]. Mouse models have demonstrated that the outcome of Nocardia infection (clearance vs. abscess formation) appears to be dependent on inoculum size, stage of Nocardia growth, and immune status of the mouse [14]. While Nocardia is a well-described cause of persistent neutrophilic meningitis [1,3], it generally occurs in immunocompromised patients following dissemination from a pulmonary focus. Here we present a case of chronic Nocardia meningitis in an immunocompetent host. We speculate that in our patient, a low inoculum of Nocardia entered the immune-privileged subdural space from the basilar skull fracture, resulting in prolonged infection with persistent neutrophilic inflammation without abscess formation.

This case highlights a number of the unique features of Nocardia meningitis including the acute to chronic presentation with fevers, headache and meningismus with persistent neutrophilic pleocytosis, hypoglycorrhachia and elevated CSF protein levels. The largest review of Nocardia meningitis includes 21 cases in which a predisposing condition was noted in $75 \%$ of cases, neutrophilic pleocytosis in $85 \%$, hypoglycorrhachia in $64 \%$ and elevated protein in $61 \%$. Forty-three percent of the cases also had a brain abscess and one case had a basilar skull fracture. Diagnosis was often difficult and often delayed likely contributing to high mortality. In this series, mortality was $57 \%$ overall [8] which is similar to other smaller case series [7]. The widespread clinical application of ribosomal DNA sequencing technology (16s rDNA) may lead to earlier diagnosis resulting in improved outcomes in these difficult cases. We cannot be certain in our patient if the portal of entry was direct inoculation from the occipital wound or from a sinus defect created by the basilar skull fracture with prior nasopharyngeal colonization.

Prior to molecular phenotyping Nocardia asteroides complex was taxonomically categorized into 6 drug pattern susceptibilities [15]. It is currently thought that Nocardia cyriacigeorgica (the pathogen recovered in this case) represents Nocardia asteroides drug pattern VI [16], and is the most common Nocardia spp isolate 
found in the southern United States [16]. The organism is typically susceptible to cephalosporins, amikacin, imipenem and linezolid, while resistant to penicillin, clarithromycin and ciprofloxacin [17]. Isolates of Nocardia cyriacigeorgica have recently been reported in a variety of clinical scenarios [18-20] making this organism an important clinical pathogen, which is increasingly being reported as a source of infection in the United States [17].

\section{Conclusions}

This unique case demonstrates the importance of considering Nocardia infection as a cause of persistent neutrophilic meningitis even in immunocompetent patients. Microbiologic diagnosis can be difficult to obtain due to the organism's slow rate of growth and sensitivity to numerous antibiotics which are generally started early and empirically. Interestingly, our patient did clinically respond to treatment with meropenem, which was enough to sterilize the CSF but insufficient to fully treat the infection (as demonstrated by relapse even after a 6 + week course of therapy). Our patient eventually made a full recovery after completing 6 weeks of ceftriaxone and TMP-SMX followed by one year of therapy with TMP-SMX and doxycycline.

\section{Acknowledgements}

We would like to acknowledge Eliah Aronoff-Spencer for his contributions to patient care

\section{Author details}

${ }^{1}$ Department of Medicine, Division of Infectious Diseases, University of California, San Diego, California, USA. ${ }^{2}$ Department of Medicine, Division of Infectious Diseases, University of California, Los Angeles, California, USA

\section{Authors' contributions}

JSG was directly involved in the care and diagnosis of this patient, manuscript preparation, editing and submission. SRA was involved in patient care, diagnosis and manuscript editing. DZU contributed to patient care, diagnosis and manuscript editing. SRM contributed to diagnosis, long term follow-up and patient care, manuscript preparation and editing. All authors have read and approved the final manuscript.

\section{Competing interests}

The authors declare that they have no competing interests.

Received: 13 January 2011 Accepted: 19 May 2011

Published: 19 May 2011

\section{References}

1. Peacock JE Jr, McGinnis MR, Cohen MS: Persistent neutrophilic meningitis. Report of four cases and review of the literature. Medicine (Baltimore) 1984, 63(6):379-395.

2. Akkara Veetil BM, Yee AH, Warrington KJ, Aksamit AJ Jr, Mason TG: Aseptic meningitis in adult onset Still's disease. Rheumatol Int 2010

3. Peacock JE Jr: Persistent neutrophilic meningitis. Infect Dis Clin North Am 1990, 4(4):747-767

4. Nichols WG, Corey L, Gooley T, Davis C, Boeckh M: High risk of death due to bacterial and fungal infection among cytomegalovirus (CMV)seronegative recipients of stem cell transplants from seropositive donors: evidence for indirect effects of primary CMV infection. I Infect Dis 2002, 185(3):273-282
5. Ambrosioni J, Lew D, Garbino J: Nocardiosis: updated clinical review and experience at a tertiary center. Infection 2010, 38(2):89-97.

6. Munoz J, Mirelis B, Aragon LM, Gutierrez N, Sanchez F, Espanol M Esparcia O, Gurgui M, Domingo P, Coll P: Clinical and microbiological features of nocardiosis 1997-2003. J Med Microbio/ 2007, 56(Pt 4):545-550.

7. Al Soub H, Almaslamani M, Al Khuwaiter J, El Deeb Y, Khatab MA: Primary Nocardia meningitis in a patient without a predisposing condition: case report and review of the literature. Scand J Infect Dis 2007, 39(8):737-741.

8. Bross JE, Gordon G: Nocardial meningitis: case reports and review. Rev Infect Dis 1991, 13(1):160-165.

9. Lerner PI: Nocardiosis. Clin Infect Dis 1996, 22(6):891-903, quiz 904-895.

10. Vander Heiden T, Stahel PF, Clutter S, Price C, Peterson SL, Morgan SJ: Nocardia osteomyelitis: a rare complication after intramedullary nailing of a closed tibial shaft fracture. J Orthop Trauma 2009, 23(3):232-236.

11. Beaman BL, Black CM, Doughty F, Beaman L: Role of superoxide dismutase and catalase as determinants of pathogenicity of Nocardia asteroides: importance in resistance to microbicidal activities of human polymorphonuclear neutrophils. Infect Immun 1985, 47(1):135-141.

12. Filice GA: Resistance of Nocardia asteroides to oxygen-dependent killing by neutrophils. J Infect Dis 1983, 148(5):861-867.

13. Filice GA: Inhibition of Nocardia asteroides by neutrophils. J Infect Dis 1985, 151(1):47-56.

14. Beaman BL: Nocardia as a pathogen of the brain: mechanisms of interactions in the murine brain-a review. Gene 1992, 115(1-2):213-217.

15. Wallace RJ Jr, Steele LC, Sumter G, Smith JM: Antimicrobial susceptibility patterns of Nocardia asteroides. Antimicrob Agents Chemother 1988, 32(12):1776-1779.

16. Conville PS, Witebsky FG: Organisms designated as Nocardia asteroides drug pattern type $\mathrm{VI}$ are members of the species Nocardia cyriacigeorgica. J Clin Microbiol 2007, 45(7):2257-2259.

17. Schlaberg R, Huard RC, Della-Latta P: Nocardia cyriacigeorgica, an emerging pathogen in the United States. J Clin Microbiol 2008, 46(1):265-273

18. Alp E, Yildiz O, Aygen B, Sumerkan B, Sari I, Koc K, Couble A, Laurent F Boiron $P$, Doganay M: Disseminated nocardiosis due to unusual species: two case reports. Scand J Infect Dis 2006, 38(6-7):545-548.

19. Barnaud G, Deschamps C, Manceron V, Mortier E, Laurent F, Bert F, Boiron P, Vinceneux P, Branger C: Brain abscess caused by Nocardia cyriacigeorgica in a patient with human immunodeficiency virus infection. J Clin Microbio/ 2005, 43(9):4895-4897.

20. Elsayed S, Kealey A, Coffin CS, Read R, Megran D, Zhang K: Nocardia cyriacigeorgica septicemia. J Clin Microbiol 2006, 44(1):280-282

\section{Pre-publication history}

The pre-publication history for this paper can be accessed here: http://www.biomedcentral.com/1471-2334/11/136/prepub

doi:10.1186/1471-2334-11-136

Cite this article as: Green et al:: Persistent Neutrophilic Meningitis in an Immunocompetent Patient after Basilar Skull Fracture: Case Report. BMC Infectious Diseases 2011 11:136.

\section{Submit your next manuscript to BioMed Central and take full advantage of:}

- Convenient online submission

- Thorough peer review

- No space constraints or color figure charges

- Immediate publication on acceptance

- Inclusion in PubMed, CAS, Scopus and Google Scholar

- Research which is freely available for redistribution 